doi: 10.32620/oikit.2021.92.09

УДК 62.822

А. Б. Жукевич, О. А. Жукевич

\title{
Синтез систем управління електроприводами 3 ковзними режимами
}

\author{
Національний аерокосмічний університет ім. М. Є. Жуковського \\ «Харківський авіаційний інститут» \\ *Харківський національний університет радіоелектроніки
}

\begin{abstract}
Синтез систем управління електроприводами методами теорії аналітичного конструювання оптимальних регуляторів (АКОР) дозволяє отримати структури 3 ковзними режимами, які вирізняються високими динамічними показниками і властивістю грубості відносно до параметричних і координатних збурень [1]. Одночасно, через наявність релейного регулятора, що працює в ковзному режимі, такі системи виходять нелінійними, що описуються диференціальними рівняннями з розривною правою частиною. У той же час однією з проблем АКОР є проблема вибору вагових коефріцієнтів оптимізуючих фуннкціоналів, яка так і не отримала прийнятного для проектувальників вирішення: вибір цих коефіцієнтів заздалегідь не визначено фрізично зрозумілими рекомендаціями. Якщо в лінійному випадку підбір вагових коефріцієнтів квадратичних критеріїв можна організувати шляхом моделювання на EOM перехідних процесів в замкнутій лінійній системі, то в нелінійному випадку такий підхід $€$ математично некоректним. Це пояснюється тим загальновідомим фактом, що для нелінійних систем принцип суперпозиції $€$ неправдивим, а їх поведінка на відміну від лінійних істотно залежить від початкових умов. У зв'язку з цим, вибравши в результаті моделювання нелінійної системи на ЕОМ ті чи інші доцільні на перший погляд вагові коефіцієнти відповідних критеріїв, можна отримати практично непрацездатну нелінійну систему, оскільки при інших початкових умовах її поведінка може якісно змінитися. Дивно, але цей загальновідомий фракт в літературі затушовується, хоча його вплив на поведінку синтезованих систем є принциповим [2].

Існують методи побудови оптимальних систем, які базуються на синтезі регуляторів на основі задання бажаних показників якості перехідних процесів 3 урахуванням відомої поведінки системи з визначеним характеристичним рівнянням. У статті обґрунтовано методику пошуку коефіцієнтів зворотних зв'язків релейного регулятора з умови забезпечення динамічних характеристик системи, заданих бажаним характеристичним рівнянням.
\end{abstract}

Ключові слова: АКОР, релейний регулятор, квадратичний функціонал, перехідні процеси, ковзний режим, гіперплощина ковзання, електропривод.

\section{Вступ}

Постановка задачі. Будемо розглядати системи управління, що описуються лінійними диференціальними рівняннями 3 постійними коефріцієнтами n-го порядку:

$$
\frac{d \mathbf{x}}{d t}=\mathbf{A x}+\mathbf{m} u
$$

де $\quad \mathbf{x}=\left\|x_{1}(t) \quad x_{2}(t) \quad \cdots \quad x_{n-1}(t) \quad x_{n}(t)\right\|$-вектор-стовпець змінних стану збуреного руху системи, елементи якого являють собою відхилення реальних координат системи від їх заданих значень; 
$\mathbf{A}=\left\|a_{i j}\right\|_{n n}-$ квадратна матриця коефіцієнтів, розмір якої визначається порядком системи диференціальних рівнянь об'єкта управління;

$$
\begin{aligned}
\mathbf{m} & =\left\|\begin{array}{llll}
0 & \cdots & 0 & m
\end{array}\right\|^{\mathbf{T}}-\text { вектор-стовпець постійних коефіцієнтів; } \\
u & =u(t) \text { - скалярна функція управління. }
\end{aligned}
$$

Необхідно знайти характеристичне рівняння замкнутої системи управління при релейному регуляторі, який описується законом

$$
u=-\operatorname{sign}\left(\mathbf{b}^{\mathbf{T}} \mathbf{x}\right) \text {, }
$$

де $\mathbf{b}=\left\|b_{1} \cdots \quad b_{n-1} \quad b_{n}\right\|^{\mathbf{T}}-$ вектор-стовпець коефіцієнтів зворотних зв'язків.

Передбачається, що в такій системі управління існує ковзний режим. Узагальнену структурну схему системи управління показано на рис. 1.

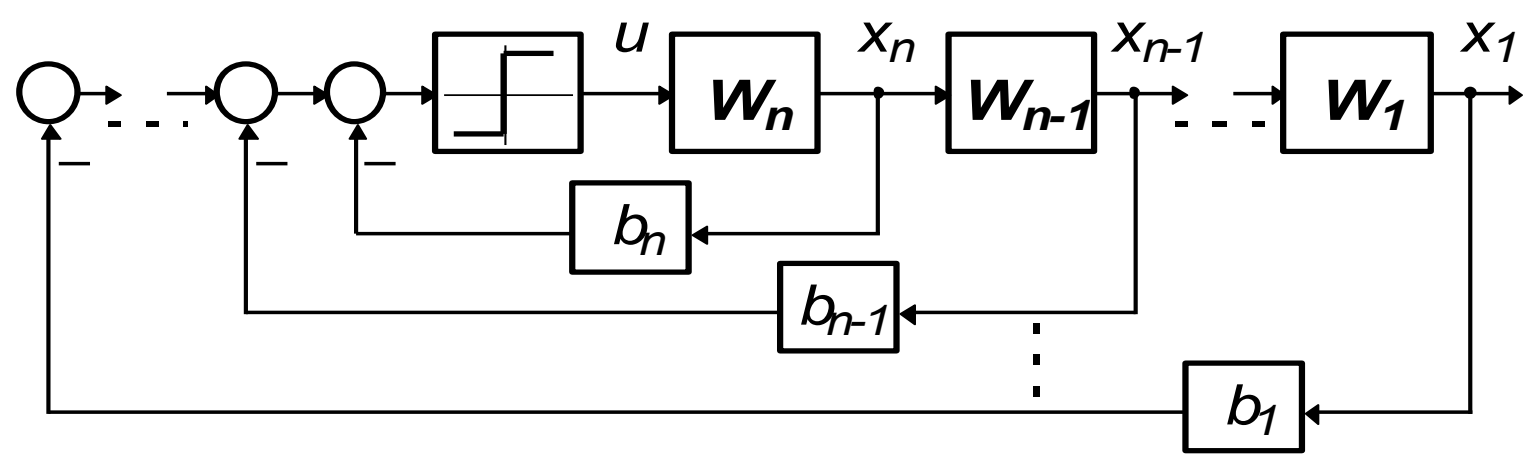

Рис. 1 Узагальнена структурна схема системи управління

\section{1 Розв'язання задачі пошуку коефіцієнтів релейного регулятора}

Для систем управління електроприводами характерна відсутність прямих паралельних каналів регулювання. В цьому випадку в матриці $\mathbf{A}$ із системи (1) елементи, які стоять вище першої наддіагонали, дорівнюють нулю, тобто $a_{i, j+1}=0$ при $i<j$ і матриця $\mathbf{A}$ має такий вигляд:

$$
\mathbf{A}=\left\|\begin{array}{lllll}
a_{11} & a_{12} & 0 & \ldots & 0 \\
a_{21} & a_{22} & a_{23} & \ldots & 0 \\
\cdots & \ldots & \ldots & \ldots & \cdots \\
\cdots & \ldots & \ldots & \ldots & a_{n-1, n} \\
a_{n 1} & a_{n 2} & a_{n 3} & \cdots & a_{n n}
\end{array}\right\| .
$$

Відповідно до методики довизначення лінійних диференціальних рівнянь з розривною правою частиною А. Ф. Філіппова [3] можна довизначити систему (1) в ковзному режимі, використавши рівняння гіперплощини перемикання, рівняння якої находиться під знаком sign:

$$
\mathbf{b}^{\mathbf{T}} \mathbf{x}=0 \text { або в розгорнутому вигляді } b_{1} x_{1}+b_{2} x_{2}+\cdots+b_{n} x_{n}=0 \text {. }
$$


Звідки виділимо внутрішню координату системи управління

$$
x_{n}=-\frac{1}{b_{n}}\left(b_{1} x_{1}+b_{2} x_{2}+\cdots+b_{n-1} x_{n-1}\right) .
$$

Для зниження порядку системи, згідно з [4] необхідно в рівняння руху системи (1) підставити значення отриманої координати $x_{n}$ з попереднього виразу.

У результаті стає можливим знизити порядок системи диференціальних рівнянь (1). Довизначена в ковзному режимі система є однорідною:

$$
\frac{d \mathbf{f}}{d t}=\mathbf{S} \mathbf{f}
$$

де $\mathbf{E}=\left\|x_{1}(t) \quad x_{2}(t) \quad \cdots \quad x_{n-1}(t)\right\|^{\mathbf{T}}$ - вектор-стовпець змінних стану, розмірність якого $(\mathrm{n}-1)$, що не містить координату $x_{n}$.

Коефіцієнти отриманої замкнутої системи (4) знаходять за фрормулою

$$
s_{i j}=a_{i j}-b_{j} \frac{a_{i n}}{b_{n}} .
$$

Матриця коефріцієнтів $\mathbf{S}$ при цьому має вигляд

$$
\mathbf{S}=\left\|s_{i j}\right\|_{n-1, n-1}=\left\|\begin{array}{lcccc}
a_{11} & a_{12} & 0 & \cdots & 0 \\
a_{21} & a_{22} & a_{23} & \cdots & 0 \\
\cdots & \ldots & \ldots & \cdots & \ldots \\
s_{n-1,1} & s_{n-1,2} & s_{n-1,3} & \cdots & s_{n-1, n-1}
\end{array}\right\|,
$$

а характеристичне рівняння системи (4), яке можна визначити через наведену вище матрицю

$$
\|p \mathbf{E}-\mathbf{S}\|=0
$$

де $\mathbf{E}=\operatorname{diag}\|1 \quad 1 \quad 1 \quad \cdots \quad 1\|_{n-1, n-1}$-діагональна одинична матриця.

Завдання синтезу може бути ссрормульовано таким чином: для синтезованої методами аналітичного конструювання структури регулятора (2) для об'єкта управління (1) визначити коефіцієнти зворотних зв'язків регулятора, при яких характеристичне рівняння замкнутої системи управління буде мати такий вигляд:

$$
p^{n-1}+c_{n-1} p^{n-2}+\cdots+c_{2} p+c_{1}=0
$$

У ковзному режимі система (1) лінеаризується. Структурна схема еквівалентної лінійної системи зниженого порядку наведена на рис. 2. 


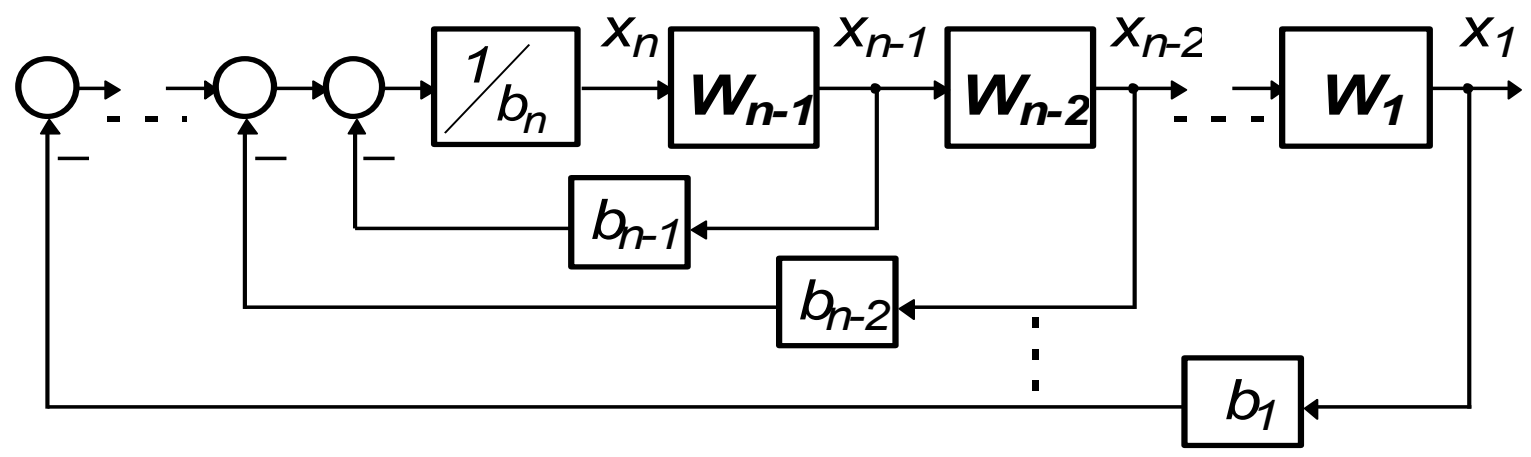

Рис. 2 Лінеаризована система (1) зниженого порядку

Тут внутрішню координату $x_{n}$ можна навести як нову функцію управління $v$, що є лінійною комбінацією всіх інших координат системи, тобто

$$
x_{n}=v\left(x_{1}, \cdots, x_{n-1}\right)=-\frac{b_{1}}{b_{n}} x_{1}-\frac{b_{2}}{b_{n}} x_{2}-\cdots-\frac{b_{n-1}}{b_{n}} x_{n-1} .
$$

Так як в законі управління (2) важливий тільки знак лінійної комбінації $\mathbf{b}^{\mathbf{T}} \mathbf{x}$, то можна задати значення одного $з$ коефіцієнтів зворотних зв'язків регулятора, при цьому пропорційно змінивши всі інші:

$$
\mathbf{b}=\left\|b_{1} \cdots b_{n-1} \quad 1\right\|^{\mathbf{T}} .
$$

Тоді функція управління довизначеної системи матиме вигляд

$$
x_{n}=v\left(x_{1}, \cdots, x_{n-1}\right)=-b_{1} x_{1}-b_{2} x_{2}-\cdots-b_{n-1} x_{n-1}=-\boldsymbol{G}^{\mathbf{T}} \mathbf{E},
$$

де $\mathbf{\epsilon}=\left\|\begin{array}{llll}b_{1} & b_{2} & \cdots & b_{n-1}\end{array}\right\|^{\mathbf{T}}$-вектор коесріцієнтів зворотних зв'язків, що не містить елемента $b_{n}$.

У результаті цього рух системи (1) у ковзному режимі може бути описано диференціальним рівнянням

$$
\frac{d \mathbf{f}}{d t}=\mathbf{G} \mathbf{f}+\mathbf{q} v,
$$

в якому матриця $\mathbf{G}$ розмірності $(n-1, n-1)$ виходить з матриці $\mathbf{A}$ рівняння (1) шляхом викреслення з неї елементів останнього рядка і останнього стовпця, а вектор постійних коефріцієнтів $\mathbf{q}$ розмірності $(n-1)$ складається з елементів $a_{\text {in }}$ останнього стовпця матриці А за винятком елемента $a_{n n}$.

Для систем електроприводу відповідно до матриці (3) 


$$
\mathbf{G}=\left\|\begin{array}{llllc}
a_{11} & a_{12} & 0 & \ldots & 0 \\
a_{21} & a_{22} & a_{23} & \ldots & 0 \\
\cdots & \ldots & \ldots & \ldots & \ldots \\
\ldots & \ldots & \ldots & \ldots & a_{n-2, n-1} \\
a_{n-1,1} & a_{n-1,2} & a_{n-1,3} & \cdots & a_{n-1, n-1}
\end{array}\right\|, \quad \mathbf{q}=\|\|\left\|\begin{array}{c}
0 \\
0 \\
\ldots \\
0 \\
a_{n-1, n}
\end{array}\right\| .
$$

Перетворемо систему рівнянь (8) до канонічного вигляду:

$$
\mathbf{E}=\mathbf{T y} ; \quad \mathbf{G}=\mathbf{T G}^{\circ} \mathbf{T}^{\mathbf{1}} ; \quad \mathbf{q}=\mathbf{T q}^{\circ}
$$

де $\mathbf{y}=\mathbf{y}(t)$ - вектор-стовпець нових змінних стану системи;

$\mathbf{T}=\left\|t_{i j}\right\|_{n n}-$ матриця лінійного перетворення до канонічного вигляду.

Тоді можна записати

$$
\begin{aligned}
& \frac{d \mathbf{y}}{d t}=\mathbf{G}^{\circ} \mathbf{y}+\mathbf{q}^{\circ} v ; \\
& \mathbf{G}^{\circ}=\mathbf{T}^{-1} \mathbf{G T}=\left\|\begin{array}{rrrrr}
0 & 1 & 0 & \cdots & 0 \\
0 & 0 & 1 & \cdots & 0 \\
\cdots & \ldots & \cdots & \cdots & 0 \\
-g_{0} & -g_{1} & -g_{2} & \cdots & -g_{n-2}
\end{array}\right\|, \quad \mathbf{q}^{\circ}=\mathbf{T}^{-1} \mathbf{q}=\left\|\begin{array}{c}
0 \\
\cdots \\
0 \\
1
\end{array}\right\| .
\end{aligned}
$$

Тут $g_{0}, g_{1}, \cdots, g_{n-2}$-коефіцієнти характеристичного полінома розімкнутої системи (8):

$$
\|p \mathbf{E}-\mathbf{G}\|=p^{n-1}+g_{n-2} p^{n-2}+\cdots+g_{1} p+g_{0} .
$$

Скористаємося властивістю системи диференціальних рівнянь канонічного вигляду, який полягає у тому, що якщо диференціальне рівняння замкнутої системи управління (4) записано в канонічній формі, то подати його можливо у вигляді

$$
\begin{gathered}
\frac{d \mathbf{y}}{d t}=\mathbf{S}^{\circ} \mathbf{y}, \\
\mathbf{S}^{\circ}=\left\|s_{i j}^{\circ}\right\|_{n-1, n-1}=\left\|\begin{array}{ccccc}
0 & 1 & 0 & \cdots & 0 \\
0 & 0 & 1 & \cdots & 0 \\
\cdots & \cdots & \cdots & \cdots & \cdots \\
-\tilde{n}_{1} & -\tilde{n}_{2} & -\tilde{n}_{3} & \cdots & -\tilde{n}_{n-1}
\end{array}\right\| .
\end{gathered}
$$

де

Коефіцієнти останнього рядка матриці $\mathbf{S}^{\circ}$ збігаються з коефіцієнтами бажаного характеристичного рівняння (6), взятими зі зворотним знаком. умови

Дійсно, характеристичне рівняння замкнутої системи (12) знайдемо 3 


$$
\left\|p \mathbf{E}-\mathbf{S}^{\circ}\right\|=\left\|\begin{array}{ccccc}
p & -1 & 0 & \cdots & 0 \\
0 & p & -1 & \cdots & 0 \\
\cdots & \cdots & \cdots & \cdots & \cdots \\
\tilde{n}_{1} & \tilde{n}_{2} & \tilde{n}_{3} & \cdots & \tilde{n}_{n-1}+p
\end{array}\right\|=0 .
$$

Визначник з рівняння (13) легко розкривається за елементами останнього рядка, тому що алгебраїчними доповненнями відповідних елементів $€$ трикутні визначники, що дорівнюють як відомо [5], добутку елементів головної діагоналі. 3 одного боку алгебраїчне доповнення елемента $k$-го стовпчика, таким чином, буде $p^{k-1}$, так що характеристичне рівняння замкнутої системи (12)

$$
\left\|p \mathbf{E}-\mathbf{S}^{\circ}\right\|=p^{n-1}+\tilde{n}_{n-1} p^{n-2}+\cdots+\tilde{n}_{2} p+\tilde{n}_{1}=0
$$

збігається з бажаним характеристичним рівнянням (6).

3 іншого боку, функція управління $v$ в рівнянні (10) являє собою лінійну форму координат системи

$$
v\left(y_{1}, y_{2}, \cdots, y_{n-1}\right)=-h_{1} y_{1}-h_{2} y_{2}-\cdots h_{n-1} y_{n-1}=-\mathbf{h}^{\mathbf{T}} \mathbf{y},
$$

де $\mathbf{h}=\left\|h_{1} \quad h_{2} \quad \cdots \quad h_{n-1}\right\|^{\mathbf{T}}$-вектор-стовпець коефіцієнтів зворотних зв'язків закону управління в системі з фазовими координатами.

Це дозволяє провести перетворення системи (10) і отримати

$$
\frac{d \mathbf{y}}{d t}=\mathbf{G}^{\circ} \mathbf{y}+\mathbf{q}^{\circ} v=\mathbf{G}^{\circ} \mathbf{y}-\mathbf{q}^{\circ} \mathbf{h}^{\mathbf{T}} \mathbf{y}=\left(\mathbf{G}^{\circ}-\mathbf{q}^{\circ} \mathbf{h}^{\mathbf{T}}\right) \mathbf{y},
$$

причому матриця коефріцієнтів

$$
\mathbf{G}^{\circ}-\mathbf{q}^{\circ} \mathbf{h}^{\mathbf{T}}=\left\|\begin{array}{cccc}
0 & 1 & \cdots & 0 \\
0 & 0 & \cdots & 0 \\
\cdots & \cdots & \cdots & \cdots \\
-h_{1}-g_{0} & -h_{2}-g_{1} & \cdots & -h_{n-1}-g_{n-2}
\end{array}\right\| .
$$

Порівнявши форми запису (12) і (15) еквівалентної лінійної системи, можна отримати рівняння

$$
\mathbf{h}+\mathbf{g}=\mathbf{c},
$$

де $\mathbf{g}=\left\|g_{0} \quad g_{1} \quad \cdots \quad g_{n-2}\right\|^{\mathbf{T}}-$ вектор-стовпець коефіцієнтів характеристичного полінома (11) розімкнутої системи;

$$
\mathbf{c}=\left\|\begin{array}{llll}
c_{1} & c_{2} & \cdots & c_{n-1}
\end{array}\right\|^{\mathbf{T}} \text {-вектор-стовпець коефіцієнтів бажаного }
$$
характеристичного рівняння (6) замкнутої системи.

Звідки коефріцієнти зворотних зв'язків закону управління (14) в системі канонічного виду (10) 


$$
\mathbf{h}=\mathbf{c}-\mathbf{g} .
$$

Використавши зворотне перетворення і врахувавши (16), з умови тотожності законів управління (7) і (14) визначимо коефріцієнти зворотних зв'язків для системи (8):

$$
\begin{aligned}
& -\overline{\mathbf{G}}^{\mathbf{T}} \mathbf{E}=-\left(\mathbf{c}^{\mathbf{T}}-\mathbf{g}^{\mathbf{T}}\right) \mathbf{y} ; \\
& \boldsymbol{\sigma}^{\mathbf{T}} \mathbf{E}=\left(\mathbf{c}^{\mathbf{T}}-\mathbf{g}^{\mathbf{T}}\right) \mathbf{T}^{-1} \mathbf{f} ; \\
& \hat{\mathbf{G}}^{\mathrm{T}}=\left(\mathbf{c}^{\mathrm{T}}-\mathbf{g}^{\mathrm{T}}\right) \mathbf{T}^{-1} ; \\
& \hat{\overline{\mathbf{b}}}=\left(\mathbf{T}^{-1}\right)^{\mathbf{T}}(\mathbf{c}-\mathbf{g}) .
\end{aligned}
$$

Вектор зворотних зв'язків релейного регулятора (2) початкової системи (1) виразимо через вектор Бิ таким чином:

$$
\mathbf{b}=\left\|\boldsymbol{\sigma}^{\mathbf{T}}: 1\right\|^{\mathbf{T}},
$$

тобто додається елемент, який дорівнюється одиниці.

Таким чином, якщо бажану якість системи управління для об'єкта (1) 3 релейним регулятором (2) задано як рішення однорідного диференційного рівняння з постійними коефіцієнтами

$$
x^{(n-1)}+c_{n-1} \cdot x^{(n-2)}+\cdots c_{2} \cdot \dot{x}+c_{1} \cdot x=0,
$$

то коефіцієнти зворотних зв'язків регулятора (2) знайдемо за формулами (17), (18) з використанням коефріцієнтів характеристичного полінома (11) розімкнутої системи (8).

Матрицю Т перетворення заданої системи диференціальних рівнянь до канонічного вигляду визначено методами теорії матриць [3]. В роботі [4] запропоновано метод відшукання цієї матриці 3 використанням матриці керованості. Для системи (8) матрицю Т може бути визначено як

$$
\mathbf{T}=\mathbf{K} \| \begin{array}{ccccc}
g_{1} & g_{2} & \cdots & g_{n-2} & 1 \\
g_{2} & g_{3} & \cdots & 1 & 0 \\
\cdots & \cdots & \cdots & \cdots & \cdots \\
g_{n-2} & 1 & \cdots & 0 & 0 \\
1 & 0 & \cdots & 0 & 0
\end{array}
$$

При цьому $g_{1}, g_{2}, \cdots, g_{n-1} \quad-$ коэфрфициенты характеристического багаточлена (11), а

$$
\mathbf{K}=\left\|\mathbf{q} \vdots \mathbf{G q} \vdots \mathbf{G}^{2} \mathbf{q} \vdots \cdots \vdots \mathbf{G}^{n-2} \mathbf{q}\right\|
$$

$€$ матриця керованості розміру $(n-1, n-1)$, перший стовпець якої складається з елементів вектору $\mathbf{q}$, наступний стовпець - $з$ елементів вектору $\mathbf{G q}$ i т.д. Оскільки розглянута система (8) цілком керована, тобто з любого початкового стану ії можна перевести в кінцевий стан за допомогою вхідного сигналу 
протягом кінцевого інтервалу часу, то матриця керованості $\mathbf{K}$ має ранг, який дорівнює $(n-1)$, що однозначно визначає існування оберненої матриці $\mathbf{K}^{-1}$.

Слід звернути увагу на таку обставину. Для обчислення матриці лінійного перетворення (9) до канонічного вигляду і розрахунку коефіцієнтів зворотних зв'язків регулятора (2) за фрормулами (17), (18) необхідно знати коефріцієнти характеристичного полінома (11). Відомі різні способи обчислення коефіцієнтів $q_{k}$ [3]. Можна вказати ефективний алгоритм, що також використовує матрицю керованості К [4], а саме добуток

$$
\mathbf{K}^{-1} \mathbf{G K}=\left\|\begin{array}{ccccc}
0 & 0 & \cdots & 0 & -g_{0} \\
1 & 0 & \cdots & 0 & -g_{1} \\
0 & 1 & \cdots & 0 & -g_{2} \\
\cdots & \cdots & \cdots & \cdots & \cdots \\
0 & 0 & \cdots & 1 & -g_{n-2}
\end{array}\right\|=\mathbf{F}
$$

утворює матрицю Фробеніуса $\mathbf{F}$. Елементи останнього їі стовпця являють собою взяті з протилежним знаком коефіцієнти характеристичного полінома (11). При цьому $g_{n-1}=1$.

\section{2 Приклад розрахунку коефіцієнтів системи електроприводу. Математичне моделювання системи «перетворювач-двигун»}

Як приклад розглянемо систему "перетворювач-двигун", що описується системами диференціальних рівнянь (1) з матрицями коефіцієнтів

$$
\begin{aligned}
& \mathbf{A}=\left\|\begin{array}{lll}
0 & a_{12} & 0 \\
a_{21} & a_{22} & a_{23} \\
0 & 0 & a_{33}
\end{array}\right\|, \quad \mathbf{m}=\left\|\begin{array}{c}
0 \\
0 \\
m
\end{array}\right\|, \\
& \text { де } a_{12}=\frac{R}{T_{M} c \Phi} \frac{I_{\ni}}{\omega_{\ni}} ; \quad a_{21}=\frac{c \Phi}{R T_{Я}} \frac{\omega_{\ni}}{I_{\ni}} ; \quad a_{22}=-\frac{1}{T_{\text {Я }}} \text {; } \\
& a_{23}=\frac{1}{R T_{Я}} \frac{E_{\ni}}{I_{\ni}} ; \quad a_{33}=-\frac{1}{T_{\Pi}} ; \quad m=\frac{\beta}{T_{\Pi}} \frac{U_{m}}{E_{\Pi}} ;
\end{aligned}
$$


Необхідно вибрати релейний регулятор, що забезпечує настройку відповідно до модульного оптимуму, при якому характеристичне рівняння замкнутої системи визначається виразом

$$
2 T_{\mu}^{2} p^{2}+2 T_{\mu} p+1=0
$$

де $T_{\mu}$ у системах підлеглого управління електроприводами $€$ малою некомпенсованою постійною часу.

За фрормулами (14), (15) отримуємо

$$
\mathbf{K}=\left\|\begin{array}{cc}
0 & a_{12} a_{23} \\
a_{23} & a_{22} a_{23}
\end{array}\right\| ; \quad \mathbf{K}^{-1}=\frac{1}{-a_{12} a_{23}}\left\|\begin{array}{cc}
a_{22} & -a_{12} \\
-1 & 0
\end{array}\right\| ; \quad \mathbf{F}=\mathbf{K}^{-1} \mathbf{G K}=\left\|\begin{array}{ll}
0 & a_{12} a_{23} \\
a_{23} & a_{22} a_{23}
\end{array}\right\| .
$$

Сформуємо систему (8) з коефіцієнтами

$$
\mathbf{G}=\left\|\begin{array}{ll}
0 & a_{12} \\
a_{21} & a_{22}
\end{array}\right\| ; \quad \mathbf{g}=\left\|\begin{array}{l}
0 \\
a_{23}
\end{array}\right\| .
$$

За фрормулами (20), (21) отримуємо

$$
\begin{gathered}
\mathbf{K}=\left\|\begin{array}{cc}
0 & a_{12} a_{23} \\
a_{23} & a_{22} a_{23}
\end{array}\right\| ; \quad \mathbf{K}^{-1}=\frac{1}{-a_{12} a_{23}}\left\|\begin{array}{cc}
a_{22} & -a_{12} \\
-1 & 0
\end{array}\right\| ; \quad \mathbf{F}=\mathbf{K}^{-1} \mathbf{G K}=\left\|\begin{array}{cc}
0 & a_{12} a_{23} \\
a_{23} & a_{22} a_{23}
\end{array}\right\| . \\
g_{0}=-a_{12} a_{21} ; \quad g_{1}=-a_{22} .
\end{gathered}
$$

Тоді матрицю $\mathbf{T}$ лінійного перетворення до канонічного вигляду визначається з формули (19):

$$
\mathbf{T}=\mathbf{K}\left\|\begin{array}{cc}
g_{1} & 1 \\
1 & 0
\end{array}\right\|=\left\|\begin{array}{cc}
a_{12} a_{23} & 0 \\
0 & a_{23}
\end{array}\right\| ; \quad \mathbf{T}^{-1}=\frac{1}{a_{12} a_{23}}\left\|\begin{array}{cc}
1 & 0 \\
0 & a_{12}
\end{array}\right\| .
$$

За фрормулою (17) знаходимо

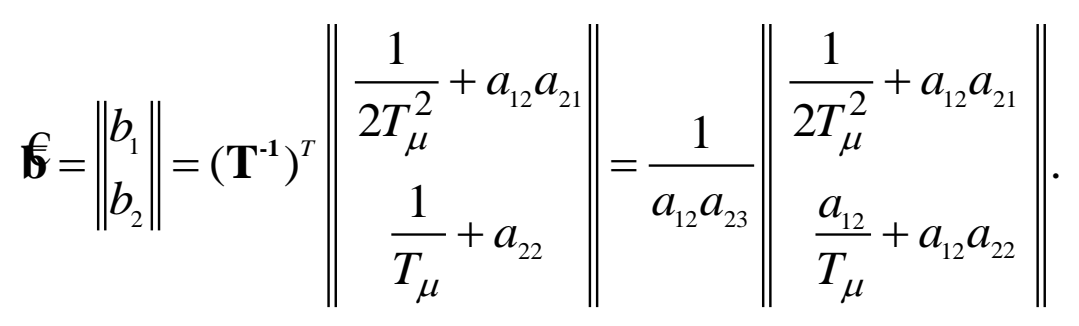

Коефіцієнти зворотних зв'язків регулятора (2) мають такі значення:

$$
b_{1}=\left(\frac{c \Phi T_{Я} T_{M}}{2 T_{\mu}^{2}}-c \Phi\right) \frac{\omega_{\ni}}{I_{\ni}} ; \quad b_{2}=\left(\frac{T_{Я}}{T_{\mu}}-1\right) R_{\Omega} \frac{I_{\ni}}{E_{\ni}} ; \quad b_{3}=1 .
$$

Отриманий закон управління в фрізичних координатах об'єкта має вигляд 


$$
\begin{aligned}
U & =U_{m} \operatorname{sign}\left[\left(b_{1}+b_{3} c \Phi\right) \omega^{*}-b_{1} \omega-b_{2} I-b_{3} E_{\Pi}\right]= \\
& =U_{m} \operatorname{sign}\left(b_{0} \omega^{*}-b_{1} \omega-b_{2} I-b_{3} E_{\Pi}\right),
\end{aligned}
$$

де $\omega^{*}-$ задане значення швидкості.

На рис. 3 наведено результати моделювання перехідного процесу пуску двигуна до швидкості $100 \mathrm{c}^{-1}$ i подальшого гальмування. При моделюванні розглядалася система з двигуном ПБВ-100L з наступними даними:

$$
\begin{aligned}
& R_{Я}=0,25 O \mu ; c \Phi=0,5 B \cdot c ; T_{я}=0,0065 c ; \\
& T_{M}=0,03 c ; T_{\Pi}=T_{\mu}=0.005 c ; \beta=6 .
\end{aligned}
$$

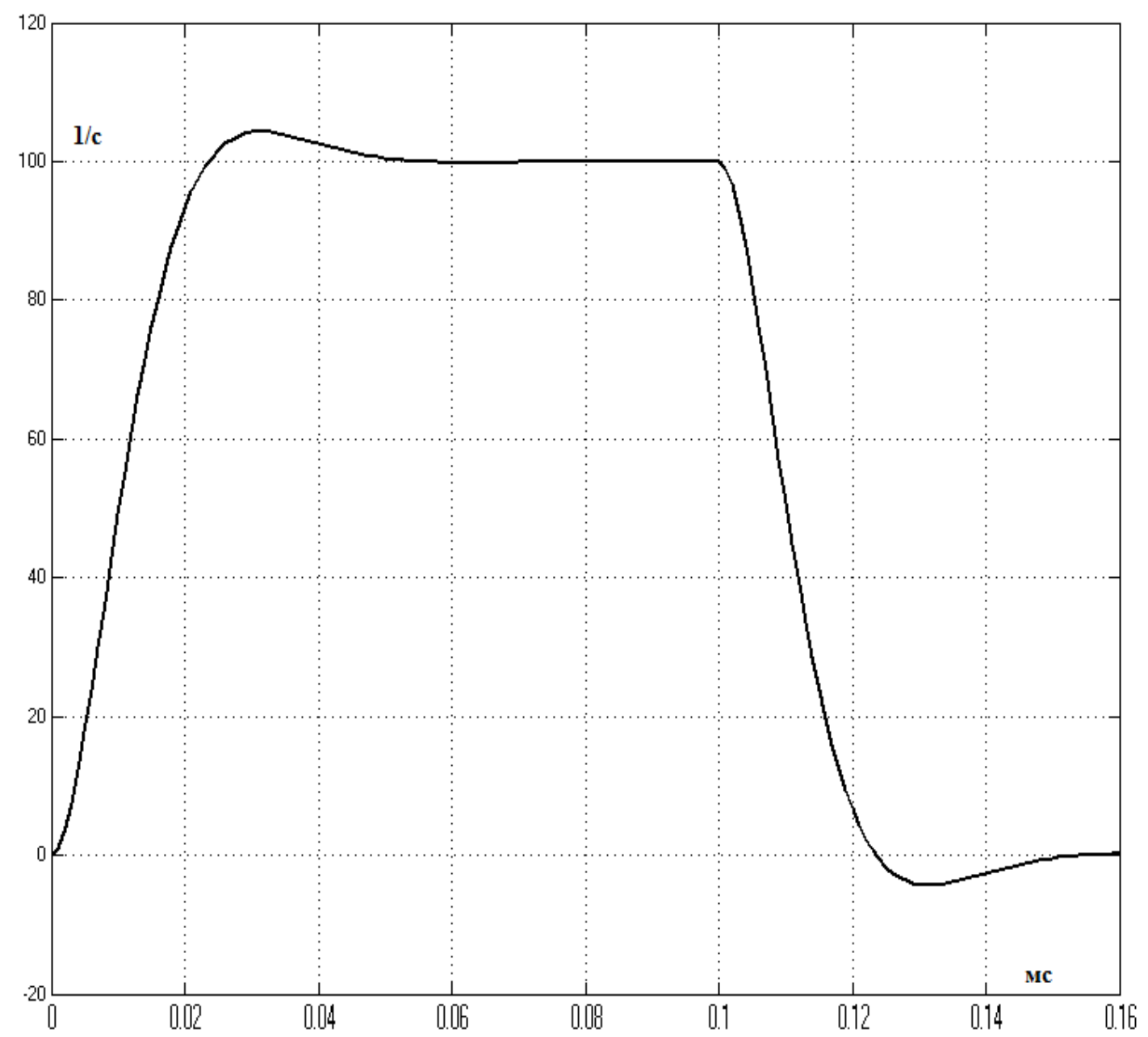

Рис. 3 Моделювання перехідних процесів пуску та гальмування двигуна з релейним регулятором

\section{Висновки}

Таким чином, запропоновано методику визначення параметрів релейного регулятора, якщо задано бажане характеристичне рівняння замкнутої системи управління. Коефіцієнти характеристичного рівняння можуть бути визначені для 
традиційних критеріїв якості на основі, наприклад, діаграми Вишеградського, кореневих методів та ін.

\section{Список літератури.}

1. Синтез электропривода, обладающего низкой чувствительностью к параметрическим возмущениям / А. Б. Зеленов, А. И. Мотченко, А. В. Садовой и др. // Изв.вузов. Электромеханика. - 1982. - №3. C.285- 291.

2. Колесников А. А. Методы АКАР и АКОР в задачах синтеза нелинейных систем управления. / А. А. Колесников, Ал. А. Колесников, А. А. Кузьменко // Мехатроника, автоматика, управление. - 2016. - Том 17. - №10. C. $657-669$.

3. Филиппов А. Ф. Диффреренциальные уравнения с разрывной правой частью. / А. Ф. Филиппов // Математический сборник - 1960 - 51(93) №1- C.99 - 128.

4. Гантмахер Ф. Р. Теория матриц. / Ф.Р.Филиппов - М.: Наука 1966. - 576 c..

\section{References}

1. Sintez jelektroprivoda, obladajushhego nizkoj chuvstvitel'nost'ju $k$ parametricheskim vozmushhenijam / A. B. Zelenov, A. I. Motchenko, A. V. Sadovoj i dr. // Izv.vuzov. Jelektromehanika. - 1982. - №3. - S.285 - 291.

2. Kolesnikov A. A. Metody AKAR i AKOR v zadachah sinteza nelinejnyh sistem upravlenija. / A. A. Kolesnikov, Al. A. Kolesnikov, A. A. Kuz'menko // Mehatronika, avtomatika, upravlenie. -2016. - Tom 17. - №10. - S. 657-669.

3. Filippov A. F. Differencial'nye uravnenija s razryvnoj pravoj chast'ju. / A. F. Filippov // Matematicheskij sbornik - 1960 - 51(93) - №1- S.99 - 128. 576 c.

4. Gantmaher F. R. Teorija matric. / F.R.Filippov - M.: Nauka - 1966. -

Надійшла до редакції 10.05.2021, розглянута на редколегії 10.05.2021.

\section{Synthesis of control systems for electric drives with sliding modes}

Synthesis of electric drive control systems by methods of the theory of analytical design of optimal controllers (ACOR) allows to obtain structures with sliding modes, which are characterized by high dynamic performance and the property of roughness relative to parametric and coordinate perturbations. At the same time, due to the presence of a relay regulator operating in sliding mode, such systems are nonlinear, described by differential equations with a discontinuous right-hand side. At the same time, one of the problems of ACOR is the problem of choosing the weights of optimizing functionals, which has not received an acceptable solution for designers: the choice of these coefficients is not predetermined by physically clear recommendations. If in the linear case the selection of weights of quadratic criteria 
can be organized by computer simulation of transients in a closed linear system, then in the nonlinear case such an approach is mathematically incorrect. This is due to the well-known fact that for nonlinear systems the principle of superposition is false, and their behavior in contrast to linear depends significantly on the initial conditions. In this regard, choosing as a result of modeling a nonlinear system on a computer those or other seemingly appropriate weights of the relevant criteria, you can get almost inoperable nonlinear system, because under other initial conditions, its behavior can change qualitatively. Surprisingly, this well-known fact in the literature is obscured, although its influence on the behavior of synthesized systems is fundamental.

There are methods for constructing optimal systems based on the synthesis of regulators based on setting the desired quality indicators of transients, taking into account the known behavior of the system with a certain characteristic equation. The article substantiates the method of finding the feedback coefficients of the relay controller from the condition of providing the dynamic characteristics of the system, given the desired characteristic equation.

Keywords: ACOR, relay regulator, quadratic functional, transients, sliding mode, sliding hyperplane, electric drive

\section{Відомості про авторів:}

Жукевич Аркадій Борисович - канд.техн.наук, доцент кафедри Систем управління літальними апаратами, Національний аерокосмічний університет ім.М.Є.Жуковського «Харківський авіаційний інститут», Харків, Україна, a.zhukevych@khai.edu.

Жукевич Олександр Аркадійович - студент, фракультет «Комп'ютерних наук», спеціальності «Штучний інтелект», Харківський Національний Університет Радіоелектроніки, Харків, Україна.

\section{About the Authors:}

Zhukevych Arkadii - Ph.D, Associate Professor, Department of Aircraft Control Systems, National Aerospace University, M.Ye.Zhukovsky "Kharkiv Aviation Institute", Kharkiv, Ukraine, a.zhukevych@khai.edu.

Zhukevych Oleksandr - student, Fakulty of Computer Systems, Specialty "Artificial Intelligence", Kharkiv National University of Radio Electronics, Kharkiv, Ukraine. 